\title{
Probing the Dynamics and the Atomic Structure of Gold Nanorods in Solution with Liquid-Cell TEM
}

\author{
Abdelali Khelfa, Caroline Byun, Christian Ricolleau, Jaysen Nelayah, Guillaume Wang and \\ Damien Alloyeau
}

Laboratoire Matériaux et Phénomènes Quantiques, CNRS / Paris-Diderot University, Paris, France.

Direct observations of nanoscale processes in liquid provide new insights into many important problems in colloidal chemistry. Therefore, the development of liquid-cell TEM has rapidly motivated intense research activities focused on nanomaterial dynamics in controlled liquid environments, including studies on the nucleation and growth mechanisms, diffusion and self-assembling phenomena, corrosion processes and the interactions between synthetic nanomaterials and biological species.[1] Nevertheless, liquid-cell TEM results are frequently put in question because the beam effects are obviously influencing the dynamics of nanomaterials in the liquid cell. Therefore, there is a crucial need to evaluate the relevance of liquid-cell TEM results by comparing them with ex situ data and to exploit low-dose electron microscopy techniques. Here, on the one hand we investigate the diffusion, colloidal stability, and dissolution processes of gold nanorods (Au NRs) in aqueous solution of tunable $\mathrm{pH}$ and ionic strength, with the view to correlate in situ nanoscale observations with ex situ macroscale optical measurements. On the other hand, we demonstrate the great potential of the STEM/NBD technique to study the atomic structure of single nanoparticles in liquid with low-dose illumination.

We have investigated the diffusion of Au NRs in the liquid-cell. In line with previous studies,[2] we confirm that nanoparticles have a sub-diffusive behavior, a few order of magnitude slower than Brownian motion, because of electrostatic interactions with the membranes. Nevertheless, the NRs can also have a convective motion when they are trapped at the liquid/gas interface.

By controlling the ionic strength and $\mathrm{pH}$ of the aqueous environment, we have induced and studied the destabilization of the colloidal solution. Remarkably, the size of the aggregates formed in the liquid-cell is in agreement with ex situ DLS measurements. We also show that the NR-NR interactions are influenced by the distribution of the organic layer at the surface of the nanoparticles.

At high ionic strength and low $\mathrm{pH}$, we have observed the dissolution of Au NRs that could explain the strong decrease of plasmon peaks measured by UV-Vis spectroscopy. As illustrated in figure 1, NRs are systematically eroded along their anisotropy axis which is in agreement with the work of Ye et al. [3]. However, we bring experimental evidences that this degradation process is mainly governed by the distribution of the organic layer at the surface of the nanoparticles that controls surface accessibility for chelating agents.

Probing the atomic structure of single nano-objects in liquid is a very important challenge for the many scientists exploiting liquid-cell TEM. Several studies have demonstrated that the formation of a hydrogen bubble within the liquid cell can be exploited to acquire HRTEM images of nanostructures in very thin liquid layers.[4] Nevertheless, such imaging conditions present several drawbacks: (i) many experiments are incompatible with the presence of such a gas/liquid interface, (ii) image resolution is limited between 2 or $3 \AA$, (iii) and more importantly high resolution imaging requires very high dose rate leading to drastic changes in the local chemistry of the liquid sample. Here we show that the STEM/NBD technique [5] (later called 4D-STEM with the development of highspeed camera) is a promising opportunity to probe the atomic structure of single nano-objects while avoiding the limitations of HRTEM. As described in figure 2, this technique consists in acquiring a low-dose STEM-HAADF image of a large area, on which the beam can then be positioned on any pixel to acquire a nano-beam diffraction (NBD) of single NRs by using a high frame rate camera (Gatan, one view). Note that structural analysis requires to use low-convergence angles to avoid the 
overlap of diffraction beams. Interestingly, in a thin liquid layer, diffraction signal can be detected with a frame rate of 300 images/second leading to a great reduction of the electron dose. We demonstrate that the number of electrons irradiating a single NR during STEM/NBD acquisition is reduced by a factor 5000 compared to the acquisition a conventional HRTEM image. Moreover, as reciprocal-space analysis is not limited by image resolution, STEM/NBD allows analyzing the atomic structure of NRs for any zone-axis orientations (figure $2 b-c$ ). These preliminary results show that STEM/NBD is a viable strategy to conduct low-dose structural analysis of single nano-objects in liquid environments.

\section{References:}

[1] F Ross, Science 350 (2015), p. 1490.

[2] A Verch et al, Langmuir 31 (2015), p. 6956.

[3] X Ye et al, Science 354 (2016), p. 874.

[4] N Ahmad et al, Advanced Structural and Chemical Imaging 2, p. 9.

[5] D Alloyeau et al, Ultramicroscopy 108 (2008), p. 656.
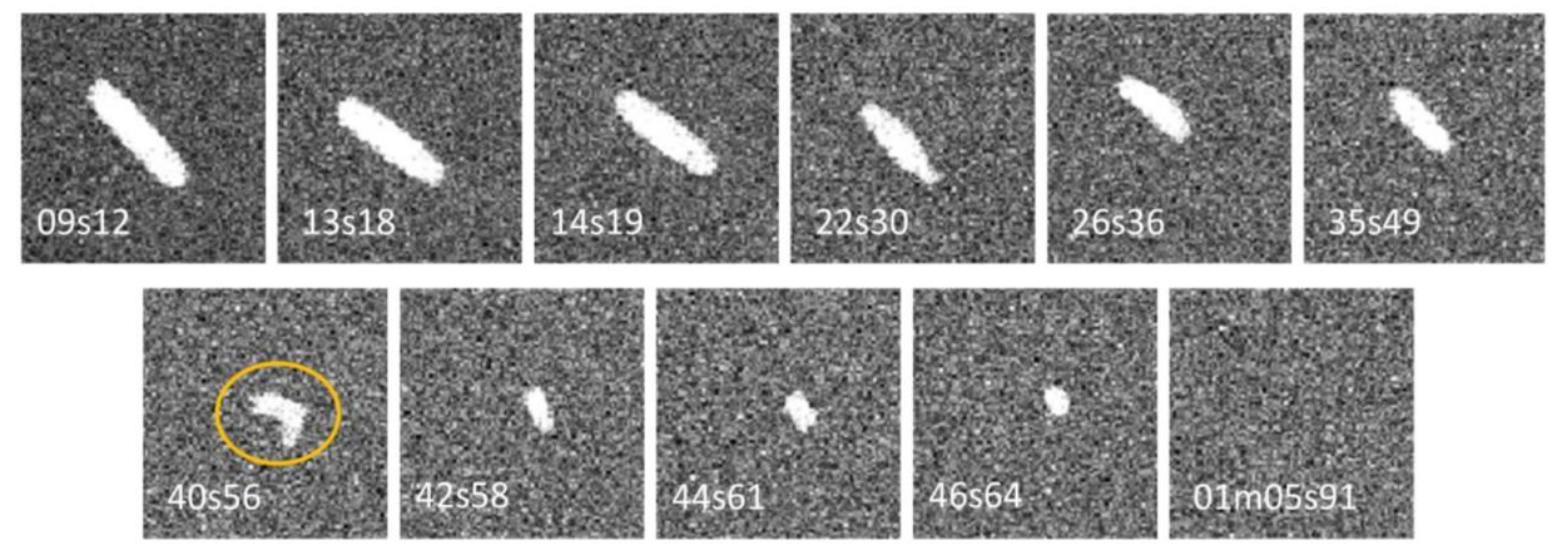

Figure 1. Image series of the dissolution of a $\mathrm{Au} \mathrm{NR}$ in an acidic saline aqueous solution $(\mathrm{NaCl}$ and $\mathrm{HCl}, \mathrm{pH}=2$ ). Acquisition time is indicated on each image and image size is $85 \mathrm{~nm}$ by $85 \mathrm{~nm}$.
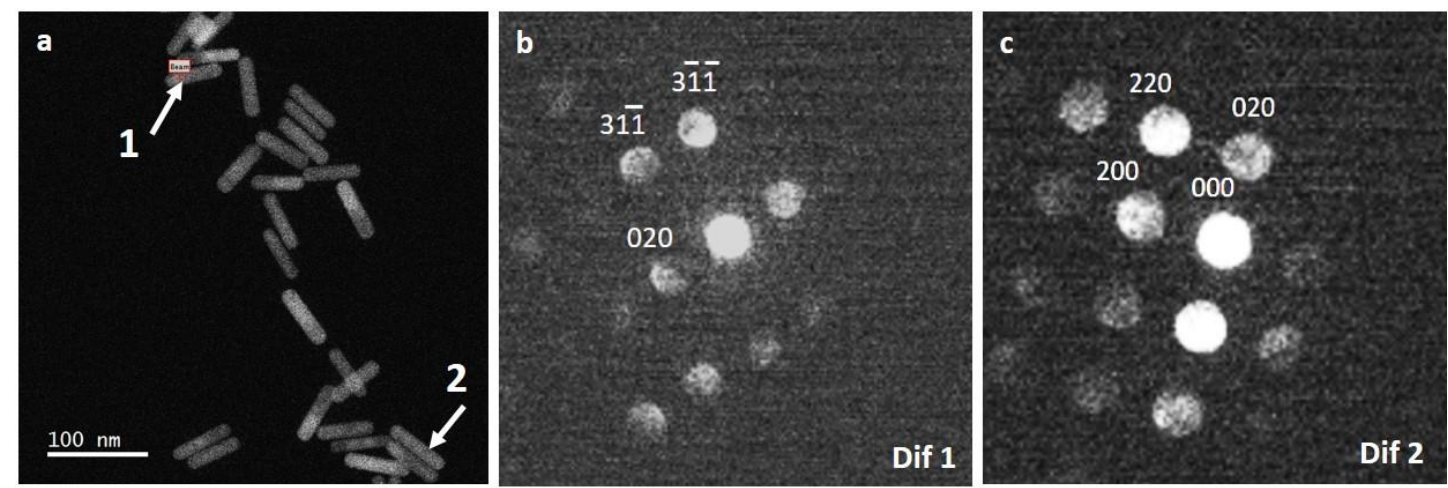

Figure 2. STEM/NBD analysis. (a) Low-dose STEM-HAADF image of Au NRs. Nanobeam diffraction acquired at the (b) position 1 on a NR oriented along the [103] direction, (c) position 2 on a NR oriented along the [001] direction. 